\title{
ASYMPTOTIC METHODS FOR MAGNETOHYDRODYNAMIC INSTABILITY
}

\author{
BY \\ MISHA VISHIK (Department of Mathematics, The University of Texas at Austin, Austin, Texas) \\ AND
}

SUSAN FRIEDLANDER (Department of Mathematics, University of Illinois at Chicago)

\begin{abstract}
A sufficient condition for instability is obtained for the linearized equations of ideal magnetohydrodynamics. The results are proved by studying the full asymptotic expansion for the evolution operator using oscillatory integrals. It is shown that the growth rate of the evolution operator is bounded from below by the growth rate of an operator given by a system of local hyperbolic PDEs.
\end{abstract}

1. Introduction. Vishik and Friedlander [17] studied the linearized stability of the Euler equations that describe the motion of an inviscid, incompressible fluid (a so-called ideal fluid). The main tool used in [17] is the full asymptotic expansion of the evolution operator (Green's function) for the Euler equation linearized about a steady flow $U(x)$. Details of the structure of the spectrum of the linearized Euler equation are unknown for general flows. An important feature of the "geometric optics" approach, applied to hyperbolic systems by Ludwig [13], Friedlander [7], and utilized in [17], is that it is not necessary to study the spectrum explicitly. Rather a lower bound is obtained for the growth rate of the evolution operator as $t \rightarrow \infty$. This lower bound is given in terms of a geometric quantity which can be considered as a "Lyapunov-type" exponent for fluid motion. It provides an effective sufficient condition for linear instability of an ideal fluid. For example, it can be used to demonstrate that any steady ideal fluid flow with exponential stretching, even along one Lagrangian trajectory, is unstable [8].

In this present paper we extend the analysis in [17] to the case of the equations of ideal magnetohydrodynamics (MHD) which govern the motion of an electrically conducting fluid. Again the approach taken is to study the full asymptotic expansion of the evolution operator for the linearized ideal MHD equations. The MHD problem is considerably more difficult than the problem for the Euler equations because the existence of a magnetic field, in addition to the velocity field, gives rise to characteristics of variable multiplicity. It is therefore necessary to introduce a new ansatz for the asymptotics that differs from the regular WKB asymptotics that was appropriate in [17] for the pure fluid equations.

Received November 29, 1995.

1991 Mathematics Subject Classification. Primary 35; Secondary 76. 
The operators that must be estimated are no longer "standard" Fourier integral operators [15]. The ansatz in question allows for separation of the quasilocal effects and the effects of the shift along the characteristics. The main result of this paper is a sufficient condition for instability (see Theorem 10.6). In the case of MHD this sufficient condition is given in terms of the growth rate of an evolution operator defined by a system of local hyperbolic PDEs. The operator acts on initial conditions as distributions along a magnetic field line. The dominant contribution to the asymptotics comes from high-frequency waves whose wave vectors are slightly tilted to the direction perpendicular to the magnetic field line.

In a recent paper Vishik [16] strengthens the results in [17] to prove that the Lyapunovtype exponent for the Euler equations discussed above determines the logarithm of the essential spectral radius for the exponentiated spectrum of the linearized Euler equations. In [16] the evolution operator is represented as a product of a pseudodifferential operator and the operator for the shift along the trajectories of the steady flow $U(x)$. We find it useful to adopt a modification of this approach for the case of MHD.

The results in this present paper are proved for the free space problem. All the analogous results are also valid for the case of periodic boundary conditions. The two sets of boundary conditions are treated in [16] and [17] for the Euler equations. For the sake of brevity we omit the proofs for the periodic MHD problem.

The sufficient condition for instability given by Theorem 10.6 is a precise mathematical formulation of the concept of local instability widely discussed in the physical literature. We note that the theorem is effective. For example, in the case where the equilibrium velocity $U(x)$ and magnetic field $H(x)$ are both axisymmetric and purely toroidal the instability criterion reduces to the calculation of the eigenvalues of an algebraic equation [9]. This can be used to reproduce "classical" results (cf. Chandrasekhar [3]) and to demonstrate "new" instabilities in classes of MHD equilibria.

There is a considerable body of work, particularly in connection with plasma physics, where the problem of MHD stability is investigated using rapidly oscillating localized perturbations to study so-called "ballooning" modes associated with the continuous spectrum (cf. Hameiri [10], Lifschitz [12]). The importance of perturbations whose wave vector is perpendicular to the magnetic field is a well-known phenomenon in MHD stability analysis. Eckhoff [6] was the first to exploit this "preferred" direction for instability by utilizing a weakly-coupled hyperbolic system. He suggested that the interaction of waves implied by the nonuniform multiplicity of characteristics may be a major cause of MHD instability. The results of this present paper, where the full asymptotic expansion of the evolution operator is constructed, has [6] as its starting point.

The following gives an outline of the material to be found in this paper. In Sec. 3 we discuss the concept of parallel transport along the trajectory of a solenoidal (divergence free) vector field. In Sec. 4 we introduce the ansatz for the formal asymptotic expansion of the evolution operator represented as an oscillatory integral. We obtain a system of non-strictly hyperbolic equations for the amplitudes. In Sec. 5 we derive estimates for solutions to this system of transport equations and show that Huygens' Principle is valid. In Sec. 6 we obtain estimates for the oscillatory integral that arises from our representation of the evolution operator. The main technical tool that we utilize is a 
variant of the Calderon-Vaillancourt inequality [2]. Refined versions of this theorem can be found in [1], [11], [14]. In Sec. 7 we prove a main technical result (Theorem 7.1 ), which justifies the asymptotics. Section 8 contains some estimates for composition formulae of pseudodifferential operators that are used in the proof of Theorem 7.1. In Sec. 9 we introduce the phase function $S$ for a high-frequency perturbation localized on a magnetic field line and we obtain an explicit formula for solving the transport equations. Section 10 contains the main result, namely the instability criterion given by Theorem 10.6 which provides a lower bound on the growth rate of the evolution operator for the MHD equations. The crucial estimate used to prove this result is given by Theorem 10.3.

2. Equations of magnetohydrodynamics. Let $U(x)$ and $H(x)$ denote an equilibrium velocity and magnetic field respectively: $x \in \mathbb{R}^{n}$. We have the following conditions describing an ideal hydromagnetic equilibrium:

$$
\begin{gathered}
-(U, \nabla) U+(H, \nabla) H-\nabla P=0 \\
\{U, H\} \equiv-(U, \nabla) H+(H, \nabla) U=0 \\
\operatorname{div} U=0, \quad \operatorname{div} H=0 .
\end{gathered}
$$

The expression on the LHS of (2.2) is a Poisson bracket and the vanishing of $\{U, H\}$ implies that the two flows generated by the two vector fields $U$ and $H$ commute. The expression $(U, \nabla) H$ denotes differentiation of $H$ along $U$.

We will study in this paper the following perturbation problem:

$$
\begin{gathered}
\dot{u}=-(U, \nabla) u-(u, \nabla) U+(h, \nabla) H+(H, \nabla) h-\nabla p \\
\dot{h}=-(u, \nabla) H-(U, \nabla) h+(h, \nabla) U+(H, \nabla) u, \\
\operatorname{div} u=0, \quad \operatorname{div} h=0 .
\end{gathered}
$$

Here $u$ is a velocity perturbation and $h$ is a magnetic field perturbation.

Adding and subtracting (2.4), (2.5) yields

$$
\begin{gathered}
(u+h)^{\circ}=-(U-H, \nabla)(u+h)-(u-h, \nabla)(U+H)-\nabla p, \\
(u-h)^{\circ}=-(U+H, \nabla)(u-h)-(u+h, \nabla)(U-H)-\nabla p, \\
\operatorname{div}(u+h)=0, \quad \operatorname{div}(u-h)=0 .
\end{gathered}
$$

We assume in this paper that $U(x)$ and $H(x)$ are $C^{\infty}$-smooth although all the results hold for the case of sufficiently smooth $U$ and $H$.

The problem (2.7) $-(2.9)$ is considered with the initial conditions

$$
u+\left.h\right|_{t=0}=u_{0}(x)+h_{0}(x), \quad u-\left.h\right|_{t=0}=u_{0}(x)-h_{0}(x) .
$$

We assume that the following estimate holds:

$$
\sup _{x \in \mathbb{R}^{n}}\left|\frac{\partial^{\alpha} H}{\partial x^{\alpha}}\right|+\sup _{x \in \mathbb{R}^{n}}\left|\frac{\partial^{\alpha} U}{\partial x^{\alpha}}\right| \leq C_{\alpha}, \quad \alpha \in \mathbb{Z}_{+}^{n} .
$$

The case where $U$ and $H$ are periodic in $x$ can be treated in a similar manner. 
3. Parallel transport. We will discuss the definition of parallel transport in slightly more generality then we are going to use in this paper. Let $M^{n}$ be an $n$-dimensional Riemannian manifold with metric denoted by $(\cdot, \cdot)$. The volume form is induced by the Riemannian metric. Let $Y$ be a $C^{1}$ solenoidal vector field on $M^{n}: \operatorname{div} Y=0$. An operator of parallel transport along the vector field $Y$ is defined as an evolution operator for the following PDE:

$$
\begin{gathered}
\dot{X}=-\nabla_{Y} X,\left.\quad X\right|_{t=0}=X_{0} \in \Gamma\left(T M^{n}\right), \\
\mathfrak{G}_{Y}(t): X_{0} \mapsto X(t) .
\end{gathered}
$$

The right-hand side of (3.1) is understood in the sense of the Levi-Civita connection induced by the metric $(\cdot, \cdot)$. We assume that the vector field $Y$ is such that $(3.2)$ defines a strongly continuous unitary group in the space $\mathcal{L}^{2}\left(M^{n}\right)$ of square-integrable vector fields on $M$. The infinitesimal operator of this group is a canonical selfadjoint extension of $X \mapsto-\nabla_{Y} X$ defined on smooth vector fields with compact support. This is obviously the case if $M^{n}=\mathbb{R}^{n}$ and $Y=U$ (or $H$ ) satisfies the inequalities (2.11). Let $g_{Y}^{t}$ : $M^{n} \rightarrow M^{n}$ be the flow on $M^{n}$ given by the vector field $Y$. Geometrically the vector $\left(\mathfrak{G}_{Y}(t) X_{0}\right)(x) \in T_{x} M^{n}$ is obtained by parallel transport in the sense of Riemannian geometry of the vector $X_{0}\left(g_{Y}^{-t} x\right) \in T_{g_{Y}^{-1}(x)} M^{n}$ along the trajectory of the flow $g_{Y}^{t}$.

4. Formal asymptotic expansion of the evolution operator. Let $G(t): \mathcal{L}_{s}^{2} \rightarrow$ $\mathcal{L}_{s}^{2}$ denote the evolution operator for $(2.7)-(2.9)$. Here

$$
\mathcal{L}_{s}^{2}=\left\{w=(u+h, u-h) \in\left(L^{2}\left(\mathbb{R}^{n}\right)\right)^{2 n} \mid \operatorname{div}(u \pm h)=0\right\} .
$$

We are going to construct the main term of an asymptotic expansion in the following form:

$$
\begin{gathered}
\left(G_{\varepsilon}(t) w_{0}\right)(x, t)=\frac{1}{2 \pi(2 \pi \varepsilon)^{n}} \int e^{i \xi(x-y) / \varepsilon-i \tau s} a(x, \xi, t, \tau, \varepsilon)\left(\mathfrak{G}_{U}^{t} \mathfrak{G}_{H}^{s} w_{0}\right)(y) d s d \tau d^{n} y d^{n} \xi \\
p=\frac{\varepsilon}{2 \pi(2 \pi \varepsilon)^{n}} \int e^{i \xi(x-y) / \varepsilon-i \tau s}\left(q(x, \xi, t, \tau, \varepsilon),\left(\mathfrak{G}_{U}^{t} \mathfrak{G}_{H}^{s} w_{0}\right)(y)\right) d s d \tau d^{n} y d^{n} \xi
\end{gathered}
$$

Here $a(x, \xi, t, \tau, \varepsilon) \in \operatorname{Mat}_{2 n \times 2 n}(\mathbb{C}), q(x, \xi, t, \tau, \varepsilon) \in \mathbb{C}^{n}$.

The operator $\mathfrak{G}_{U}^{t} \mathfrak{G}_{H}^{s}$ acts on a vector $w_{0}=\left(u_{0}-h_{0}, u_{0}+h_{0}\right)$ by components:

$$
\mathfrak{G}_{U}^{t} \mathfrak{G}_{H}^{s} w_{0} \stackrel{\text { def }}{=}\left(\mathfrak{G}_{U}^{t} \mathfrak{G}_{H}^{s}\left(u_{0}-h_{0}\right), \mathfrak{G}_{U}^{t} \mathfrak{G}_{H}^{s}\left(u_{0}+h_{0}\right)\right)
$$

where the RHS of (4.3) is understood according to (3.1), (3.2).

Suppose the LHS of (4.1), (4.2) satisfies Eqs. (2.7)-(2.9) "up to terms of order $\varepsilon$ ". Let $a=\left(\begin{array}{l}a_{+} \\ a_{-}\end{array}\right)$where $a_{ \pm} \in \operatorname{Mat}_{n \times 2 n}(\mathbb{C})$. To shorten the notation we denote the operator corresponding to an amplitude $b(x, \xi, \tau, \varepsilon)$ as

$$
\left(\mathrm{op}_{\varepsilon, H}[b] w_{0}\right)(x)=\frac{1}{2 \pi(2 \pi \varepsilon)^{n}} \int e^{i \xi(x-y) / \varepsilon-i \tau s} b(x, \xi, \tau, \varepsilon)\left(\mathfrak{G}_{H}^{s} w_{0}\right)(y) d s d \tau d^{n} y d^{n} \xi
$$


The exact class of amplitudes will be specified later. We obtain by differentiating (4.1) in time and integrating by parts using (3.1)

$$
\frac{\partial}{\partial t}\left(G_{\varepsilon}(t) w_{0}\right)=\mathrm{op}_{\varepsilon, H}\left[\dot{a}(x, \xi, t, \tau, \varepsilon)-a \circ \frac{i}{\varepsilon}(U, \xi)\right] \circ \mathfrak{G}_{U}^{t} w_{0}
$$

Here $\circ$ denotes a composition of pseudodifferential operators ( $\tau$ is fixed). Again below we will justify this formal procedure. We used here the fact that the operators $\mathfrak{G}_{U}^{t}$ and $\mathfrak{G}_{H}^{t}$ commute and the generator of the group $\mathfrak{G}_{U}^{t}$ has an $\varepsilon$-symbol $-\frac{i}{\varepsilon}(U(x), \xi)$. The RHS of (2.7)-(2.8) has the following form: $\mathrm{op}_{\varepsilon, H}[b] \circ \mathfrak{G}_{U}^{t} w_{0}$ where

$$
\begin{aligned}
b= & \left(\begin{array}{cc}
-\frac{i}{\varepsilon}(U-H, \xi) & 0 \\
0 & -\frac{i}{\varepsilon}(U+H, \xi)
\end{array}\right) \circ a \\
& -\left(\begin{array}{cc}
0 & \frac{\partial(U+H)}{\partial x} \\
\frac{\partial(U-H)}{\partial x} & 0
\end{array}\right) a-\left(\begin{array}{c}
i \xi \otimes q \\
i \xi \otimes q
\end{array}\right)+O(\varepsilon) \\
= & -\left(\begin{array}{cc}
(U-H, \nabla) & 0 \\
0 & (U+H, \nabla)
\end{array}\right) a-\left(\begin{array}{cc}
\frac{i}{\varepsilon}(U-H, \xi) & 0 \\
0 & \frac{i}{\varepsilon}(U+H, \xi)
\end{array}\right) a \\
& -\left(\begin{array}{cc}
0 & \frac{\partial(U+H)}{\partial x} \\
\frac{\partial(U-H)}{\partial x} & 0
\end{array}\right) a-\left(\begin{array}{c}
i \xi \otimes q \\
i \xi \otimes q
\end{array}\right) a+O(\varepsilon) .
\end{aligned}
$$

We transform (4.4) using the composition formula for pseudodifferential operators:

$$
\dot{a}-a \circ \frac{i}{\varepsilon}(U, \xi)=\dot{a}-\frac{i}{\varepsilon}(U, \xi) a-\sum_{|\alpha|=1} \frac{\partial^{\alpha} a}{\partial \xi^{\alpha}} \frac{\partial^{\alpha}}{\partial x^{\alpha}}(U(x), \xi)+O(\varepsilon) .
$$

We also have, since $\mathfrak{G}_{U}^{t}$ and $\mathfrak{G}_{H}^{s}$ commute (see (2.2)),

$$
\begin{aligned}
\mathrm{op}_{\varepsilon, H} & {\left[a_{ \pm}\right] \circ \mathfrak{G}_{U}^{t}\left\{(H, \nabla) w_{0}\right\} } \\
& =\frac{1}{2 \pi(2 \pi \varepsilon)^{n}} \int e^{i \xi(x-y) / \varepsilon-i \tau s} a_{ \pm}(x, \xi, t, \tau, \varepsilon)\left(\mathfrak{G}_{u}^{t} \cdot\left(-\frac{d}{d s} \mathfrak{G}_{H}^{s}\right) w_{0}\right)(y) d s d \tau d^{n} y d^{n} \xi \\
& =\mathrm{op}_{\varepsilon, H}\left[-i \tau a_{ \pm}\right] \circ \mathfrak{G}_{U}^{t} w_{0} .
\end{aligned}
$$

On the other hand, again using the composition formula, we obtain

$$
\begin{aligned}
\mathrm{op}_{\varepsilon, H} & {\left[a_{ \pm}\right] \circ \mathfrak{G}_{U}^{t} \circ(H, \nabla) } \\
=\mathrm{op}_{\varepsilon, H} & {\left[a_{ \pm} \circ \frac{i}{\varepsilon}(H, \xi)\right] \circ \mathfrak{G}_{U}^{t} } \\
& =\mathrm{op}_{\varepsilon, H}\left[\frac{i}{\varepsilon}(H, \xi) a_{ \pm}+\sum_{|\alpha|=1} \frac{\partial^{\alpha} a_{ \pm}}{\partial \xi^{\alpha}} \frac{\partial^{\alpha}}{\partial x^{\alpha}}(H(x), \xi)+O(\varepsilon)\right] \cdot \mathfrak{G}_{U}^{t}
\end{aligned}
$$

Hence from (4.7), (4.8)

$$
\begin{aligned}
\mathrm{op}_{\varepsilon, H} & {\left[\frac{i}{\varepsilon}(H, \xi) a_{ \pm}\right] \circ \mathfrak{G}_{U}^{t} } \\
= & \mathrm{op}_{\varepsilon, H}\left[-i \tau a_{ \pm}-\sum_{|\alpha|=1} \frac{\partial^{\alpha} a_{ \pm}}{\partial \xi^{\alpha}} \frac{\partial^{\alpha}}{\partial x^{\alpha}}(H(x), \xi)+O(\varepsilon)\right] \cdot \mathfrak{G}_{U}^{t}
\end{aligned}
$$


From (4.5), (4.6), (4.9) we conclude that the approximate solution given by (4.1), (4.2) is satisfied up to the order $\varepsilon$ when the following system of local PDEs holds for the amplitudes $a_{ \pm}$(and $q$ which we still have to find):

$$
\left\{\begin{aligned}
\dot{a}_{+}= & -(U-H, \nabla) a_{+}+\sum_{|\alpha|=1} \frac{\partial^{\alpha} a_{+}}{\partial \xi^{\alpha}} \frac{\partial^{\alpha}}{\partial x^{\alpha}}(U-H, \xi)-i \tau a_{+} \\
& -\frac{\partial(U+H)}{\partial x} a_{-}-i \xi \otimes q \\
\dot{a}_{-}= & -(U+H, \nabla) a_{-}+\sum_{|\alpha|=1} \frac{\partial^{\alpha} a_{-}}{\partial \xi^{\alpha}} \frac{\partial^{\alpha}}{\partial x^{\alpha}}(U+H, \xi)+i \tau a_{-} \\
& -\frac{\partial(U-H)}{\partial x} a_{+}-i \xi \otimes q .
\end{aligned}\right.
$$

It remains to find an expression for $q$. We use the main order of the solenoidality condition $(\nabla, u \pm h)=0$. Formally, this leads to

$$
\left(\xi, a_{ \pm}(x, \xi, t, \tau) \eta\right)=0, \quad \xi \in \mathbb{R}^{n} \backslash 0, \eta \in \mathbb{R}^{2 n}
$$

We claim that the following choice satisfies the condition (4.11):

$$
(q, \eta)=\frac{i}{|\xi|^{2}}\left(\xi,\left[\frac{\partial(U-H)}{\partial x} a_{+}+\frac{\partial(U+H)}{\partial x} a_{-}\right] \eta\right), \quad \eta \in \mathbb{R}^{2 n} .
$$

Proposition 4.1. Let $a_{ \pm}(x, \xi, t, \tau)$ be the solution to the following system:

$$
\left\{\begin{aligned}
\dot{a}_{ \pm}=-(U \mp H, \nabla) a_{ \pm}+\sum_{|\alpha|=1} \frac{\partial^{\alpha} a_{ \pm}}{\partial \xi^{\alpha}} \frac{\partial^{\alpha}}{\partial x^{\alpha}}(U \mp H, \xi) \mp i \tau a_{ \pm} \\
-\frac{\partial(U \pm H)}{\partial x} a_{\mp}+\frac{\xi \otimes \xi}{|\xi|^{2}}\left(\frac{\partial(U-H)}{\partial x} a_{+}+\frac{\partial(U+H)}{\partial x} a_{-}\right) \\
(x, \xi, t, \tau) \in \mathbb{R}^{n} \times \mathbb{R}^{n} \backslash 0 \times \mathbb{R} \times \mathbb{C}
\end{aligned}\right.
$$

with initial conditions

$$
\left\{\begin{array}{l}
a_{+}(x, \xi, 0, \tau)=\left(1_{n}-\frac{\xi \otimes \xi}{|\xi|^{2}}, 0_{n}\right) \in \operatorname{Mat}_{n \times 2 n}(\mathbb{C}), \\
a_{-}(x, \xi, 0, \tau)=\left(0_{n}, 1_{n}-\frac{\xi \otimes \xi}{|\xi|^{2}}\right) \in \operatorname{Mat}_{n \times 2 n}(\mathbb{C}) .
\end{array}\right.
$$

Then (4.11) holds.

Proof. Note that the term

$$
(U \mp H, \nabla) a_{ \pm}-\sum_{|\alpha|=1} \frac{\partial^{\alpha} a_{ \pm}}{\partial \xi^{\alpha}} \frac{\partial}{\partial x^{\alpha}}(U \mp H, \xi)
$$

is the derivative in the direction of a Hamiltonian vector field on $T^{*} \mathbb{R}^{n} \backslash 0$ given by the Hamiltonian $\mathcal{H}_{ \pm}(x, \xi)=(U(x) \pm H(x), \xi), x \in \mathbb{R}^{n}, \xi \in \mathbb{R}^{n} \backslash 0$. Therefore this term can be written as $\nabla_{U \mp H} a_{ \pm}$. Thus we have on the cotangent bundle $(x, \xi)$ (with removed zero 
section):

$$
\begin{aligned}
\frac{\partial}{\partial t}\left(\xi, a_{ \pm} \eta\right)= & \left(\xi, \frac{\partial}{\partial t} a_{ \pm} \eta\right)=\left(\xi,\left[-\nabla_{U \mp H} a_{ \pm} \mp i \tau a_{ \pm}-\frac{\partial(U \pm H)}{\partial x} a_{\mp}\right] \eta\right) \\
& +\left(\xi,\left[\frac{\partial(U-H)}{\partial x} a_{+}+\frac{\partial(U+H)}{\partial x} a_{-}\right] \eta\right) \\
= & -\nabla_{U \mp H}\left(\xi, a_{ \pm} \eta\right) \mp i \tau\left(\xi, a_{ \pm} \eta\right)
\end{aligned}
$$

since clearly $\nabla_{U \mp H} \xi=-\left(\frac{\partial(U \mp H)}{\partial x}\right)^{\mathrm{T}} \xi$. Thus we have obtained two hyperbolic equations for $\left(\xi, a_{ \pm} \eta\right)$ with zero initial conditions because of (4.13). It is easy to see however that this Cauchy problem has a unique solution. This proves (4.11).

5. Estimates for the system of "transport" equations. We will obtain estimates for a solution to the system (4.12) of "transport" equations. For technical reasons it is convenient to let $\tau$ be an arbitrary complex number. We also need arbitrary initial conditions. Let the vectors $b_{ \pm}(x, \xi, t)$ satisfy the following system of equations:

$$
\begin{gathered}
\dot{b}_{ \pm}=-\nabla_{U \mp H} b_{ \pm} \mp i \tau b_{ \pm}-\frac{\partial(U \pm H)}{\partial x} b_{\mp}+\frac{\xi \otimes \xi}{|\xi|^{2}}\left(\frac{\partial(U+H)}{\partial x} b_{-}+\frac{\partial(U-H)}{\partial x} b_{+}\right) \\
b_{ \pm}(x, \xi, 0)=b_{0 \pm}(x, \xi), \quad(x, \xi) \in T^{*} \mathbb{R}^{n} \backslash 0 .
\end{gathered}
$$

Proposition 5.1. Let $\tau$ be a complex number.

1. The system (5.1) has a unique solution in the spaces $\mathcal{D}\left(T^{*} \mathbb{R}^{n} \backslash 0\right), \mathcal{E}\left(T^{*} \mathbb{R}^{n} \backslash 0\right)$, $C_{\text {loc }}^{k}\left(T^{*} \mathbb{R}^{n} \backslash 0\right), k=0,1, \ldots$ The solution is analytic in $\tau$.

2. The following estimate holds:

$$
\begin{aligned}
& \sup _{\substack{(x, \xi) \in T^{*} \mathbb{R}^{n} \backslash 0, \pm,|\alpha+\beta| \leq k}}|\xi|^{\beta}\left|\frac{\partial^{|\alpha|+|\beta|+\gamma}}{\partial x^{\alpha} \partial \xi^{\beta} \partial \tau^{\gamma}} b_{ \pm}(x, \xi, t)\right| \\
& \quad \leq C_{k, \gamma} \sup _{\substack{(x, \xi) \in T^{*} \mathbb{R}^{n} \backslash 0, \pm,|\alpha+\beta| \leq k}}|\xi|^{\beta}\left|\frac{\partial^{|\alpha|+|\beta|}}{\partial x^{\alpha} \partial \xi^{\beta}} b_{0_{ \pm}}\right| e^{t(C+|\operatorname{Im} \tau|)}
\end{aligned}
$$

for appropriate constants $C_{k, \gamma}>0, C>0$.

3. Huygens' Principle: Let $\operatorname{supp} b_{0}=K \subset \subset T^{*} \mathbb{R}^{n} \backslash 0$. Then

$$
\operatorname{supp}\left(\begin{array}{l}
b_{+}(t) \\
b_{-}(t)
\end{array}\right) \subset \bigcup_{s=0}^{t} g_{U+H}^{s} g_{U-H}^{t-s} K
$$

Proof. To simplify the notation we will consider a more general system:

$$
\dot{b}=\left(\begin{array}{cc}
-\nabla_{U-H}-i \tau & 0 \\
0 & -\nabla_{U+H}+i \tau
\end{array}\right) b+M(x, \xi) b
$$

where $M(x, \xi)$ is a $C^{\infty}$ matrix that is homogeneous of degree zero defined on $T^{*} \mathbb{R}^{n} \backslash 0$ $(M(x, \rho \xi)=M(x, \xi)$ for $\rho \neq 0)$. 
1. The results of existence and uniqueness are standard and we will omit the proof.

2. We first establish the estimate (5.2) for the system with $M=0$. Let the equilibrium fields $U$ and $H$ satisfy $(2.11)$ and let $b_{0}(x, \xi) \in C_{\mathrm{loc}}^{k}\left(T^{*} \mathbb{R}^{n} \backslash 0\right)$ satisfy

$$
\left\|b_{0}\right\|_{k}=\left\|b_{0}\right\|_{B_{k}}=\sup _{\substack{(x, \xi) \in T^{*} \mathbb{R}^{n} \backslash 0 \\|\alpha+\beta| \leq k}}|\xi|^{\beta}\left|\frac{\partial^{|\alpha|+|\beta|}}{\partial x^{\alpha} \partial \xi^{\beta}} b_{0}(x, \xi)\right|<\infty .
$$

Consider

$$
\dot{b}=-\nabla_{U \pm H} b \pm i \tau b,\left.\quad b\right|_{t=0}=b_{0} .
$$

We will prove (5.2) using induction in $k$. When $k=0$, integrating along the characteristics leads to the estimate

$$
\|b(t)\|_{0} \leq\left\|b_{0}\right\|_{0} e^{t|\operatorname{Im} \tau|} .
$$

Assume the estimate is valid for $l<k$, i.e.,

$$
\|b(t)\|_{\ell} \leq C_{\ell}\left\|b_{0}\right\|_{\ell} e^{t(C+|\operatorname{Im} \tau|)}, \quad b_{0} \in B_{l} .
$$

Let $b_{0} \in B_{k}$. Differentiating (5.5) with respect to $x_{i}$ gives

$$
\frac{\partial}{\partial x_{i}} \dot{b}=-\nabla_{U \pm H} \frac{\partial}{\partial x_{i}} b \pm i \tau \frac{\partial b}{\partial x_{i}}-\nabla_{\partial(U \pm H) / \partial x_{i}} b .
$$

Hence from Duhamel's formula

$$
\left\|\frac{\partial b(t)}{\partial x_{i}}\right\|\left\|_{k-1} \leq\right\| \frac{\partial b_{0}}{\partial x_{i}}\left\|_{k-1} C_{k-1} e^{t(C+|\operatorname{Im} \tau|)}+\int_{0}^{t} C_{k-1} e^{(t-\tau)(C+|\operatorname{Im} \tau|)} C\right\| b(\tau) \|_{k} d \tau .
$$

Differentiating (5.5) with respect to $\xi^{i}$ gives

$$
\begin{aligned}
|\xi| \frac{\partial \dot{b}}{\partial \xi^{i}}=- & \nabla_{U \pm H}\left(|\xi| \frac{\partial b}{\partial \xi^{i}}\right) \pm i \tau\left(|\xi| \frac{\partial b}{\partial \xi^{i}}\right) \\
& +\sum_{j}\left[|\xi| \frac{\partial b}{\partial \xi^{j}} \frac{\partial(U \pm H)}{\partial x_{j}}-\frac{\partial|\xi|}{\partial \xi^{j}} \frac{\partial(U \pm H, \xi)}{\partial x_{j}} \frac{\partial b}{\partial \xi^{i}}\right] .
\end{aligned}
$$

Again, from Duhamel's formula

$$
\left\||\xi| \frac{\partial b(t)}{\partial \xi^{i}}\right\|\left\|_{k-1} \leq\right\||\xi| \frac{\partial b_{0}}{\partial \xi^{i}}\left\|_{k-1} C_{k-1} e^{t(C+|\operatorname{Im} \tau|)}+\int_{0}^{t} C_{k-1} e^{(t-\tau)(C+|\operatorname{Im} \tau|)} C\right\| b(\tau) \|_{k} d \tau
$$

From (5.8) and (5.9) we obtain the estimate

$$
\|b(t)\|_{k} \leq C C_{k-1}\left\|b_{0}\right\|_{k} e^{t(C+|\operatorname{Im} \tau|)}+C \int_{0}^{t} C_{k-1} e^{(t-\tau)(C+|\operatorname{Im} \tau|)}\|b(\tau)\|_{k} d \tau .
$$

Hence, Gronwall's Lemma yields the result

$$
\|b(t)\|_{k} \leq C_{k}\left\|b_{0}\right\|_{k} e^{t(C+|\operatorname{Im} \tau|)} .
$$


We have established an estimate of the type of (5.2) in the case $M=0$ where the systems for $b_{+}$and $b_{-}$are uncoupled. We make use of this estimate for the general case where $M \neq 0$. We apply Duhamel's formula to (5.3) to obtain

$$
\|b(t)\|_{k} \leq C_{k}\left\|b_{0}\right\|_{k} e^{t(C+|\operatorname{Im} \tau|)}+C_{k} \int_{0}^{t} C_{1} e^{(t-\tau)(C+|\operatorname{Im} \tau|)}\|b(\tau)\|_{k} d \tau
$$

where $C_{1}$ is the norm of multiplication by $M(x, \xi)$ in $B_{k}$. Applying Gronwall's Lemma, we obtain the estimate (5.2) for $\gamma=0$. Differentiating (5.3) with respect to $\tau$ and using this estimate for $\gamma=0$, we prove by induction the general case of (5.2).

3. The proof of Huygens' Principle is again standard. It is particularly easy to prove in our case. Consider (5.1) written in the form (5.3). The first term on the RHS defines a unitary group in $\left(L^{2}\left(T^{*} \mathbb{R}^{n}\right)\right)^{2 n} .^{1}$ In particular, this defines canonically the domain of the operator $C=\left(\begin{array}{cc}-\nabla_{U-H} & 0 \\ 0 & \nabla_{U+H}\end{array}\right)$ in this space. Multiplication by $M(x, \xi)$ is a bounded operator $M$ in $\left(L^{2}\left(T^{*} \mathbb{R}^{n}\right)\right)^{2 n}$. Therefore we can apply the Trotter product formula

$$
e^{t(C+M)} b=\lim _{m \rightarrow \infty}\left(e^{t C / m} e^{t M / m}\right)^{m} b, \quad b \in D(C) .
$$

Now $e^{t M / m}$ obviously does not change the support while

$$
\operatorname{supp} e^{t C / m} b \subset \bigcup_{s=0}^{t / m} \mathfrak{G}_{U+H}^{s} \mathfrak{G}_{U-H}^{(t / m)-s} \operatorname{supp} b, \quad b \in D(C) .
$$

Hence applying this argument $m$ times yields Huygens' Principle.

\section{Estimates for $\mathrm{op}_{H}[a]$.}

TheOREM 6.1. Let $a(x, \xi, \tau) \in C^{\infty}\left(\mathbb{R}^{n} \times \mathbb{R}^{n} \times \mathbb{C}\right)$ be holomorphic in $\tau$. Suppose the following inequality holds:

$$
\left|\frac{\partial^{\alpha+\beta}}{\partial \xi^{\alpha} \partial x^{\beta}} a(x, \xi, \tau)\right| \leq C_{\alpha \beta} e^{\kappa|\operatorname{Im} \tau|} \lambda_{\xi}^{\rho(|\beta|-|\alpha|)}
$$

for $\max (|\alpha|,|\beta|) \leq[n / 2]+1$. Here $\kappa \geq 0$ is a fixed number, $\lambda_{\xi}=\left(1+|\xi|^{2}\right)^{1 / 2}$ and $0 \leq \rho<1$. Let $\Lambda=-(H, \nabla)$ be considered as a closed unbounded operator in $L^{2}\left(\mathbb{R}^{n}\right)$. Let

$$
\|f\|_{D(\Lambda)}=\left(\|f\|_{L^{2}\left(\mathbb{R}^{n}\right)}^{2}+\|\Lambda f\|_{L^{2}\left(\mathbb{R}^{n}\right)}^{2}\right)^{1 / 2} \quad \forall f \in D(\Lambda)
$$

be the graph norm of a vector in the domain $D(\Lambda)$ of $\Lambda$. Then op ${ }_{H}[a]$ defined on $\mathcal{D}\left(\mathbb{R}^{n}\right)$ could be extended to a bounded operator $D(\Lambda) \rightarrow L^{2}\left(\mathbb{R}^{n}\right)$ :

$$
\left\|\mathrm{op}_{H}[a] f\right\|_{\mathcal{L}\left(D(\Lambda), L^{2}\left(\mathbb{R}^{n}\right)\right)} \leq\left(\sum_{|\alpha|,|\beta| \leq[n / 2]+1} C_{\alpha \beta}\right)\|f\|_{D(\Lambda)} \quad \forall f \in D(\Lambda) .
$$

Proof. Let $f \in \mathcal{D}\left(\mathbb{R}^{n}\right)$. We have

$$
\left(\mathrm{op}_{H}[a] f\right)(x)=\frac{1}{(2 \pi)^{n+1}} \int a(x, \xi, \tau) e^{i \xi(x-y)-i \tau s}\left(\mathfrak{G}_{H}^{s} f\right)(y) d^{n} y d^{n} \xi d s d \tau .
$$

\footnotetext{
${ }^{1}$ There is a natural Liouville measure on $T^{*} \mathbb{R}^{n}$ defined by the symplectic structure.
} 
Let $T=\kappa+1$. We choose $\chi \in C^{\infty}(\mathbb{R})$ so that $\chi=1$ on $[-1,1]$ and $\chi=0$ outside $[-2,2]$. According to the Paley-Wiener-Schwartz theorem (cf. [4]) the distribution $\mathcal{F}_{\tau \rightarrow s} a(x, \xi, \tau)$ vanishes outside $[-\kappa, \kappa]$. Hence,

$$
\begin{aligned}
\left(\mathrm{op}_{H}[a] f\right)(x) & \frac{1}{(2 \pi)^{n+1}} \int a(x, \xi, \tau) e^{i \xi(x-y)-i \tau s} \chi\left(\frac{s}{T}\right)\left(\mathfrak{G}_{H}^{s} f\right)(y) d^{n} y d^{n} \xi d s d \tau \\
= & \frac{1}{(2 \pi)^{n+1}} \int a(x, \xi, \tau) e^{i \xi(x-y)-i \tau s} \frac{1}{1+i \tau}\left(i d+\frac{d}{d s}\right)\left\{\chi\left(\frac{s}{T}\right) \mathfrak{G}_{H}^{s} f(y)\right\} d^{n} y d^{n} \xi d s d \tau \\
= & \frac{1}{2 \pi} \int_{-\infty}^{\infty} d \tau \frac{1}{1+i \tau} \frac{1}{(2 \pi)^{n}} \int a(x, \xi, \tau) e^{i \xi(x-y)} e^{-i \tau s} \\
& \left\{\chi\left(\frac{s}{T}\right)\left(\mathfrak{G}_{H}^{s}((i d-(H, \nabla)) f)\right)(y)+\frac{1}{T} \chi^{\prime}\left(\frac{s}{T}\right)\left(\mathfrak{G}_{H}^{s} f\right)(y)\right\} d^{n} y d^{n} \xi d s \\
= & \frac{1}{2 \pi} \int_{-\infty}^{\infty} d \tau \frac{1}{1+i \tau} \mathrm{op}[a(\tau)] \mathcal{F}_{s \rightarrow \tau}\left\{\chi\left(\frac{s}{T}\right) \mathfrak{G}_{H}^{s}(i d-(H, \nabla)) f+\frac{1}{T} \chi^{\prime}\left(\frac{s}{T}\right) \mathfrak{G}_{H}^{s} f\right\}
\end{aligned}
$$

Therefore, from Minkowski's inequality and Plancherel's identity,

$$
\begin{aligned}
& \left\|\operatorname{op}_{H}[a] f\right\|_{L^{2}\left(\mathbb{R}^{n}\right)} \\
& \quad \leq C \sup _{\tau}\|\operatorname{op}[a(\tau)]\|_{\mathcal{L}^{2}\left(\mathbb{R}^{n}\right)}\left(\int_{-\infty}^{\infty} \frac{1}{1+\tau^{2}} d \tau\right)^{1 / 2} \\
& \quad\left\|s \mapsto \chi\left(\frac{s}{T}\right) \mathfrak{G}_{H}^{s}(i d-(H, \nabla)) f+\frac{1}{T} \chi^{\prime}\left(\frac{s}{T}\right) \mathfrak{G}_{H}^{s} f\right\|_{L^{2}\left(-T, T ; L^{2}\left(\mathbb{R}^{n}\right)\right)} \\
& \quad \leq C \sup _{\tau}\|\operatorname{op}[a(\tau)]\|_{\mathcal{L}\left(L^{2}\left(\mathbb{R}^{n}\right)\right)}\|f\|_{D(\Lambda)} .
\end{aligned}
$$

Applying a variant of the Calderon-Vaillancourt inequality [11] to the right side of (6.4) yields (6.2).

7. Approximating the Green's function. We assume in the sequel that $n=3$, which will slightly simplify the exposition. We denote by $A_{ \pm}$the solution to the system (4.12) with the following initial condition:

$$
\begin{aligned}
& A_{+}(x, \xi, 0, \tau)=\left(1_{n}-\frac{\xi \otimes \xi}{|\xi|^{2}}, 0_{n}\right)\left(1-\chi\left(\frac{\xi}{\sqrt{\varepsilon}}\right)\right), \\
& A_{-}(x, \xi, 0, \tau)=\left(0_{n}, \mathbf{1}_{n}-\frac{\xi \otimes \xi}{|\xi|^{2}}\right)\left(1-\chi\left(\frac{\xi}{\sqrt{\varepsilon}}\right)\right) .
\end{aligned}
$$

Let also $A=\left(\begin{array}{l}A_{+} \\ A_{-}\end{array}\right) \in \operatorname{Mat}_{2 n \times 2 n}(\mathbb{C})$. Here the cutoff function $\chi(\xi) \in \mathcal{D}\left(\mathbb{R}^{n}\right)$ depends on $|\xi|$ only and

$$
\chi(\xi)=\left\{\begin{array}{ll}
1, & |\xi| \leq \frac{1}{2} \\
0, & |\xi| \geq \frac{2}{3}
\end{array} \quad, \quad 0 \leq \chi(\xi) \leq 1\right.
$$


We define the following functional spaces:

$$
\begin{aligned}
& L^{2}=\left(L^{2}\left(\mathbb{R}^{n}\right)\right)^{n}, \quad L_{s}^{2}=L^{2} \cap\{w \mid \operatorname{div}=0\} \\
& \mathcal{L}^{2}=L^{2} \oplus L^{2} \\
& \mathcal{L}_{s}^{2}=L_{s}^{2} \oplus L_{s}^{2} .
\end{aligned}
$$

Let $G_{\varepsilon}^{s}(t): \mathcal{L}_{s}^{2} \rightarrow \mathcal{L}_{s}^{2}$ be constructed using the amplitudes $A_{ \pm}$:

$$
\begin{aligned}
\left(G_{\varepsilon}^{s}(t) w_{0}\right)(x) & =\left(\begin{array}{cc}
\nabla_{x} \times & 0 \\
0 & \nabla_{x} \times
\end{array}\right) \frac{\varepsilon}{(2 \pi)(2 \pi \varepsilon)^{n}} \int e^{i \xi(x-y) / \varepsilon-i \tau s} \\
& \left(\begin{array}{cc}
\frac{i \xi}{|\xi|^{2}} \times & 0 \\
0 & \frac{i \xi}{|\xi|^{2}} \times
\end{array}\right)\left(\begin{array}{l}
A_{+}(x, \xi, t, \tau) \\
A_{-}(x, \xi, t, \tau)
\end{array}\right)\left(\mathfrak{G}_{H}^{s} \mathfrak{G}_{U}^{t} w_{0}\right)(y) d y d \xi d s d \tau \\
& \stackrel{\text { def }}{\equiv} \operatorname{op}_{H}^{s}[A] \mathfrak{G}_{U}^{t} w_{0} .
\end{aligned}
$$

We also denote by $G(t): \mathcal{L}_{s}^{2} \rightarrow \mathcal{L}_{s}^{2}$ the evolution operator for (2.7)-(2.8), i.e.,

$$
G(t) w_{0}=w(t)
$$

Theorem 7.1. For any $w_{0} \in D(\Lambda)$ and $T>0$,

$$
\| G_{\varepsilon}^{s}(t) w_{0}-G(t) \circ \text { op }[1-\chi(\sqrt{\varepsilon} \xi)] w_{0}\left\|_{\mathcal{L}_{s}^{2}} \leq C \sqrt{\varepsilon}\right\| w_{0} \|_{D(\Lambda)}
$$

where the constant $C$ is uniform with respect to $t \in[0, T]$.

Proof. Let

$$
L=\left(\begin{array}{ll}
\pi & 0 \\
0 & \pi
\end{array}\right) \cdot\left(\begin{array}{cc}
(U-H, \nabla) & \frac{\partial(U+H)}{\partial x} \\
\frac{\partial(U-H)}{\partial x} & (U+H, \nabla)
\end{array}\right)
$$

where $\pi: L^{2} \rightarrow L_{s}^{2}$ is the Weyl orthogonal projector onto the subspace of solenoidal vector fields in $L^{2}$. Let $w_{0} \in \mathcal{D}\left(\mathbb{R}^{n}\right)$. We will estimate

$$
\left\|\left(\frac{\partial}{\partial t}+L\right)\left(G_{\varepsilon}^{s}(t) w_{0}-G(t) \circ \mathrm{op}[1-\chi(\sqrt{\varepsilon} \xi)] w_{0}\right)\right\|_{\mathcal{L}^{2}}=\left\|\left(\frac{\partial}{\partial t}+L\right) G_{\varepsilon}^{s}(t) w_{0}\right\|_{\mathcal{L}^{2}} .
$$

Let $q \in \mathbb{C}^{2 n}$ be defined as follows:

$$
(q(x, \xi, t, \tau), \eta)=\left(\frac{i \xi}{|\xi|^{2}},\left(\frac{\partial(U-H)}{\partial x} A_{+}+\frac{\partial(U+H)}{\partial x} A_{-}\right) \eta\right) \quad \forall \eta \in \mathbb{R}^{2 n} .
$$

Since $\pi$ is an orthogonal projection,

$$
\begin{aligned}
& \left\|\left(\frac{\partial}{\partial t}+L\right) G_{\varepsilon}^{s}(t) w_{0}\right\|_{\mathcal{L}^{2}} \\
& \quad \leq\left\|\frac{\partial}{\partial t} G_{\varepsilon}^{s}(t) w_{0}+\left(\begin{array}{cc}
(U-H, \nabla) & \frac{\partial(U+H)}{\partial x} \\
\frac{\partial(U-H)}{\partial x} & (U+H, \nabla)
\end{array}\right) G_{\varepsilon}^{s}(t) w_{0}+\left(\begin{array}{cc}
\nabla_{x} & \text { op }_{H}[q] \circ \mathfrak{G}_{U}^{t} w_{0} \\
\nabla_{x} & \text { op }_{H}[q] \circ \mathfrak{G}_{U}^{t} w_{0}
\end{array}\right)\right\|_{\mathcal{L}^{2}} .
\end{aligned}
$$


We have

$$
\begin{aligned}
& \frac{\partial}{\partial t} G_{\varepsilon}^{s}(t) w_{0}=\mathrm{op}_{H}^{s}[\dot{A}] \mathfrak{G}_{U}^{t} w_{0}-\mathrm{op}_{H}^{s}[A]\left(U, \nabla_{x}\right) \mathfrak{G}_{U}^{t} w_{0} \\
& =\operatorname{op}_{H}[\dot{A}] \mathfrak{G}_{U}^{t} w_{0}-\mathrm{op}_{H}[A]\left(U, \nabla_{x}\right) \mathfrak{G}_{U}^{t} w_{0} \\
& +\varepsilon \operatorname{op}_{H}\left[\left(\begin{array}{cc}
\nabla_{x} \times \cdot & 0 \\
0 & \nabla_{x} \times
\end{array}\right)\left(\begin{array}{cc}
\frac{i \xi \times \cdot}{|\xi|^{2}} & 0 \\
0 & \frac{i \xi \times \cdot}{|\xi|^{2}}
\end{array}\right) \dot{A}\right] \mathfrak{G}_{U}^{t} w_{0}
\end{aligned}
$$

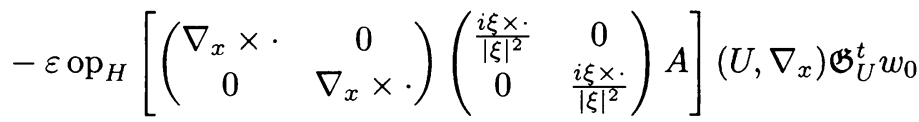

$$
\begin{aligned}
& =\mathrm{op}_{H}\left[-\left(\begin{array}{cc}
\nabla_{U-H}+i \tau & \frac{\partial(U+H)}{\partial x} \\
\frac{\partial(U-H)}{\partial x} & \nabla_{U+H}-i \tau
\end{array}\right) A-\left(\begin{array}{c}
i \xi \otimes q \\
i \xi \otimes q
\end{array}\right)\right] \mathfrak{G}_{U}^{t} w_{0} \\
& \text { - } \mathrm{op}_{H}[A]\left(U, \nabla_{x}\right) \mathfrak{G}_{U}^{t} w_{0}+\varepsilon(\cdots) \text {. }
\end{aligned}
$$

Also,

$$
G_{\varepsilon}^{s}(t) w_{0}=\operatorname{op}_{H}[A] \mathfrak{G}_{U}^{t} w_{0}+\varepsilon \mathrm{op}_{H}\left[\left(\begin{array}{cc}
\nabla_{x} \times \cdot & 0 \\
0 & \nabla_{x} \times
\end{array}\right)\left(\begin{array}{cc}
\frac{i \xi}{|\xi|^{2}} \times \cdot & 0 \\
0 & \frac{i \xi}{|\xi|^{2}} \times \cdot
\end{array}\right) A\right] \mathfrak{G}_{U}^{t} w_{0}
$$

Thus the expression on the right side of (7.9) equals

$$
\begin{aligned}
& \operatorname{op}_{H}\left[-\left(\begin{array}{cc}
\nabla_{U-H}+i \tau & \frac{\partial(U+H)}{\partial x} \\
\frac{\partial(U-H)}{\partial x} & \nabla_{U+H}-i \tau
\end{array}\right) A-\left(\begin{array}{c}
i \xi \otimes q \\
i \xi \otimes q
\end{array}\right)\right] \mathfrak{G}_{U}^{t} w_{0} \\
& +\left(\begin{array}{cc}
(U-H, \nabla) & \frac{\partial(U+H)}{\partial x} \\
\frac{\partial(U-H)}{\partial x} & (U+H, \nabla)
\end{array}\right) \operatorname{op}_{H}[A] \mathfrak{G}_{U}^{t} w_{0} \\
& +\mathrm{op}_{H}\left[\left(\begin{array}{c}
i \xi \otimes q \\
i \xi \otimes q
\end{array}\right)\right] \mathfrak{G}_{U}^{t} w_{0} \\
& -\mathrm{op}_{H}[A]\left(U, \nabla_{x}\right) \mathfrak{G}_{U}^{t} w_{0} \\
& +\varepsilon \operatorname{op}_{H}\left[\left(\begin{array}{cc}
\nabla_{x} \times & 0 \\
0 & \nabla_{x} \times
\end{array}\right)\left(\begin{array}{cc}
\frac{i \xi}{|\xi|^{2}} \times \cdot & 0 \\
0 & \frac{i \xi \times \cdot}{|\xi|^{2}}
\end{array}\right) \dot{A}\right] \mathfrak{G}_{U}^{t} w_{0} \\
& -\varepsilon \operatorname{op}_{H}\left[\left(\begin{array}{cc}
\nabla_{x} \times \cdot & 0 \\
0 & \nabla_{x} \times \cdot
\end{array}\right)\left(\begin{array}{cc}
\frac{i \xi}{|\xi|^{2}} \times & 0 \\
0 & \frac{i \xi}{|\xi|^{2}} \times \cdot
\end{array}\right) A\right]\left(U, \nabla_{x}\right) \mathfrak{G}_{U}^{t} w_{0} \\
& +\varepsilon\left(\begin{array}{cc}
(U-H, \nabla) & \frac{\partial(U+H)}{\partial x} \\
\frac{\partial(U-H)}{\partial x} & (U+H, \nabla)
\end{array}\right) \operatorname{op}_{H}\left[\left(\begin{array}{cc}
\nabla_{x} \times \cdot & 0 \\
0 & \nabla_{x} \times \cdot
\end{array}\right)\left(\begin{array}{cc}
\frac{i \xi}{|\xi|^{2}} \times & 0 \\
0 & \frac{i \xi}{|\xi|^{2}} \times \cdot
\end{array}\right) A\right] \mathfrak{G}_{U}^{t} w_{0} \\
& +\varepsilon \mathrm{op}_{H}\left(\begin{array}{c}
\nabla_{x} q \\
\nabla_{x} q
\end{array}\right) \mathfrak{G}_{U}^{t} w_{0} \stackrel{\text { def }}{=} \mathcal{R} \in \mathcal{L}^{2}
\end{aligned}
$$


We have from (7.11)-(7.18)

$$
\begin{aligned}
\mathcal{R}= & \operatorname{op}_{H}\left[-\left(\begin{array}{cc}
\nabla_{U-H}+i \tau & 0 \\
0 & \nabla_{U+H}-i \tau
\end{array}\right) A\right] \mathfrak{G}_{U}^{t} w_{0} \\
+ & \left(\begin{array}{cc}
(U-H, \nabla) & 0 \\
0 & (U+H, \nabla)
\end{array}\right) \mathrm{op}_{H}[A] \mathfrak{G}_{U}^{t} w_{0} \\
& -\operatorname{op}_{H}[A]\left(U, \nabla_{x}\right) \mathfrak{G}_{U}^{t} w_{0}+O(\varepsilon) .
\end{aligned}
$$

We will deal with the $O(\varepsilon)$ terms later. To transform the $O(1)$ part of $\mathcal{R}$ we integrate by parts with respect to $s$ and use the composition formula given in Lemmas 8.1 and 8.2 and Theorem 6.1 to get

$$
\|\mathcal{R}\|_{\mathcal{L}^{2}} \leq C \sqrt{\varepsilon}\left\|\mathfrak{G}_{U} w_{0}\right\|_{D(\Lambda)}+\|O(\varepsilon)\|_{\mathcal{L}^{2}}
$$

We use here the fact that $A$ vanishes on the neighborhood of the zero section of $T^{*} \mathbb{R}^{n}$ of radius $C \sqrt{\varepsilon}$. This follows from Huygens' principle ( $C$ is uniform with respect to $t \in[0, T])$.

To estimate the $O(\varepsilon)$-terms we note that by Theorem 6.1

$$
\begin{aligned}
& \|O(\varepsilon)\|_{\mathcal{L}^{2}} \leq C \sqrt{\varepsilon}\left\|\mathfrak{G}_{U} w_{0}\right\|_{D(\Lambda)} \\
& +\varepsilon \|-\mathrm{op}_{H}\left[\left(\begin{array}{cc}
\nabla_{x} \times \cdot & 0 \\
0 & \nabla_{x} \times
\end{array}\right)\left(\begin{array}{cc}
\frac{i \xi}{|\xi|^{2}} \times \cdot & 0 \\
0 & \frac{i \xi}{|\xi|^{2}} \times \cdot
\end{array}\right)\left(\begin{array}{cc}
\nabla_{U-H}+i \tau & 0 \\
0 & \nabla_{U+H}-i \tau
\end{array}\right) A\right] \mathfrak{G}_{u}^{t} w_{0} \\
& \quad-\mathrm{op}_{H}\left[\left(\begin{array}{cc}
\nabla_{x} \times \cdot & 0 \\
0 & \nabla_{x} \times \cdot
\end{array}\right)\left(\begin{array}{cc}
\frac{i \xi}{|\xi|^{2}} \times \cdot & 0 \\
0 & \frac{i \xi}{|\xi|^{2}} \times \cdot
\end{array}\right) A\right](U, \nabla) \mathfrak{G}_{U}^{t} w_{0} \\
& +\left(\begin{array}{cc}
(U-H, \nabla) & 0 \\
0 & (U+H, \nabla)
\end{array}\right) \mathrm{op}_{H}\left[\left(\begin{array}{cc}
\nabla_{x} \times \cdot & 0 \\
0 & \nabla_{x} \times \cdot
\end{array}\right)\left(\begin{array}{cc}
\frac{i \xi}{|\xi|^{2}} \times & 0 \\
0 & \frac{i \xi}{|\xi|^{2}} \times \cdot
\end{array}\right) A\right] \mathfrak{G}_{U}^{t} w_{0} \|_{\mathcal{L}^{2}} .
\end{aligned}
$$

Again, using the composition formula from Lemmas 8.1 and 8.2 and Theorem 6.1 we get the same estimate $C \sqrt{\varepsilon}\left\|\mathfrak{G}_{U} w_{0}\right\|_{D(\Lambda)}=C \sqrt{\varepsilon}\left\|w_{0}\right\|_{D(\Lambda)}$ for the remaining term in the right side of (7.23). From (7.8)

$$
\left(\frac{\partial}{\partial t}+L\right)\left(G_{\varepsilon}^{s}(t) w_{0}-G(t) \circ \mathrm{op}[1-\chi(\sqrt{\varepsilon} \xi)] w_{0}\right)=h(t) \in \mathcal{L}^{2}
$$

where

$$
\|h(t)\|_{\mathcal{L}^{2}} \leq C \sqrt{\varepsilon}\left\|w_{0}\right\|_{D(\Lambda)} .
$$

Let $w_{1}(t)=G_{\varepsilon}^{s}(t) w_{0}, w_{2}(t)=G(t) \mathrm{op}[1-\chi(\sqrt{\varepsilon} \xi)] w_{0}$. We multiply both sides of (7.24) by $\left(w_{1}-w_{2}\right)$ and integrate by parts. Using the fact that both $w_{1}, w_{2}$ are solenoidal we obtain

$$
\frac{1}{2} \frac{d}{d t}\left\|w_{1}-w_{2}\right\|_{\mathcal{L}^{2}}^{2} \leq C\left\|w_{1}-w_{2}\right\|_{\mathcal{L}^{2}}^{2}+C \sqrt{\varepsilon}\left\|w_{0}\right\|_{D(\Lambda)}\left\|w_{1}-w_{2}\right\|_{\mathcal{L}^{2}}
$$

Using the fact that $w_{1}(0)=w_{2}(0)$ from (7.26) and a Gronwall type of estimate we get

$$
\left\|w_{1}(t)-w_{2}(t)\right\|_{\mathcal{L}^{2}} \leq C \sqrt{\varepsilon} t\left\|w_{0}\right\|_{D(\Lambda)}
$$

where the constant $C$ is uniform with respect to $t \in[0, T]$. 


\section{Some estimates for the composition formula.}

Lemma 8.1. Let $A(x, \xi, \tau) \in C^{\infty}\left(\mathbb{R}^{n} \times \mathbb{R}^{n} \times \mathbb{C}\right)$ be holomorphic in $\tau$ and satisfy the following estimate:

$$
\left|\frac{\partial^{|\alpha+\beta|}}{\partial x^{\alpha} \partial \xi^{\beta}} A(x, \xi, \tau)\right| \leq C_{\alpha \beta}(1+|\xi|)^{m-|\beta|} e^{\kappa|\operatorname{Im} \tau|}
$$

and let $B(x, \xi) \in C^{\infty}\left(\mathbb{R}^{n} \times \mathbb{R}^{n}\right)$ satisfy the estimate

$$
\left|\frac{\partial^{|\alpha+\beta|}}{\partial x^{\alpha} \partial \xi^{\beta}} B(x, \xi)\right| \leq D_{\alpha \beta}(1+|\xi|)^{s-|\beta|} .
$$

Then, for any $N \geq \max (1, m+s)$,

$$
\begin{aligned}
\left\|\mathrm{op}[B] \circ \mathrm{op}_{H}[A]-\mathrm{op}_{H}\left[\sum_{|\gamma| \leq N-1} \frac{1}{\gamma !}\left(\frac{\partial}{\partial \xi}\right)^{\gamma} B\left(\frac{1}{i} \frac{\partial}{\partial x}\right)^{\gamma} A\right]\right\|_{\mathcal{L}\left(D(\Lambda), \mathcal{L}^{2}\right)} \\
\leq C(m, s, N, n)\left(\sum_{\substack{|\alpha| \leq 2[n / 2]+2 \\
\mid \beta \leq[n / 2]+1}} C_{\alpha \beta}\right)\left(\sum_{\substack{|\alpha| \leq[n / 2]+1 \\
N \leq|\beta| \leq 3 \mid n / 2]+3+N}} D_{\alpha \beta}\right) .
\end{aligned}
$$

Proof. The proof given in Vishik [16] applies where the reference to the CalderonVaillancourt inequality should be replaced by a reference to Theorem 6.1 .

Lemma 8.2. Let $A$ be as in Lemma 8.1 and let $B(x, \xi)=i(Y(x), \xi)$ where $Y=H$ or $U$. Then for any $N \geq \max (1, m+1)$,

$$
\begin{aligned}
& \left\|\operatorname{op}_{H}[A] \circ \mathrm{op}[B]-\mathrm{op}_{H}\left[\sum_{\gamma \leq N-1} \frac{1}{\gamma !}\left(\frac{\partial}{\partial \xi}\right)^{\gamma} A\left(\frac{1}{i} \frac{\partial}{\partial x}\right)^{\gamma} B\right]\right\|_{\mathcal{L}\left(D(\Lambda), \mathcal{L}^{2}\right)} \\
& \quad \leq C(m, N, n)\left(\sum_{\substack{|\alpha| \leq[n / 2]+1 \\
N \leq|\beta| \leq 3[n / 2]+3+N}} C_{\alpha \beta}\right) .
\end{aligned}
$$

Proof. Pull $(Y, \nabla)$ through $\mathfrak{G}_{U}^{t}$ and $\mathfrak{G}_{H}^{s}$ using the fact that they commute. Then apply the argument in Lemma 8.2 of [16] to the composition of a pseudodifferential operator with a symbol $A(x, \xi, \tau)$ for a fixed $\tau$ that is in class $S_{1,0}^{m}$ and another one with symbol $i(Y(x), \xi) \in S_{1,0}^{1}$.

REMARK. The lemma obviously generalizes to polynomials of $(H, \nabla)$ and $(U, \nabla)$. The composition formula for two general operators $\mathrm{op}_{H}[A]$ and $\mathrm{op}_{H}[B]$ could be given but this goes beyond the scope of the present paper.

\section{An explicit formula for solving the amplitude equation.}

Lemma 9.1. Let $a_{ \pm}(x, \xi, t, \tau)$ be defined by (4.12), (4.13). Let $S_{0}(x), c_{0 \pm}(x)$ be chosen so that $\left(c_{0 \pm}(x), \nabla S_{0}(x)=0, \nabla S_{0}(x) \neq 0\right.$, and $(H(x), \nabla) S_{0}(x)=0$ for any $x \in \operatorname{supp} c_{0}(x)$. 
Let $S(x, t)$ be defined by

$$
\dot{S}(x, t)=-(U, \nabla) S, \quad S(x, 0)=S_{0}(x) .
$$

Consider the system

$$
\left\{\begin{aligned}
\dot{B}_{ \pm}(x, t) & =-(U \mp H, \nabla) B_{ \pm}-\frac{\partial(U \pm H)}{\partial x} B_{\mp} \\
& +\frac{\nabla S \otimes \nabla S}{|\nabla S|^{2}}\left(\frac{\partial(U-H)}{\partial x} B_{+}+\frac{\partial(U+H)}{\partial x} B_{-}\right) \\
B_{ \pm}(x, 0) & =c_{0 \pm}(x) e^{i S_{0}(x)}
\end{aligned}\right.
$$

Then, for any $(x, t)$,

$$
B_{ \pm}(x, t)=\frac{e^{i S(x, t)}}{2 \pi} \int e^{-i \tau s}\left(\begin{array}{c}
a_{+}(x, \nabla S(x, t), t, \tau) \\
a_{-}(x, \nabla S(x, t), t, \tau)
\end{array}\right)\left(\mathfrak{G}_{U}^{t} \mathfrak{G}_{H}^{s} c_{0}\right)(x) d s d \tau
$$

Proof. For $t=0$ the statement is

$$
c_{0}(x) e^{i S_{0}(x)}=\frac{e^{i S_{0}(x)}}{2 \pi} \int e^{-i \tau s}\left(\begin{array}{cc}
\mathbf{1}_{n}-\frac{\nabla S_{0}(x) \otimes \nabla S_{0}(x)}{\left|\nabla S_{0}(x)\right|^{2}} & 0_{n} \\
0_{n} & \mathbf{1}_{n}-\frac{\nabla S_{0}(x) \otimes \nabla S_{0}(x)}{\left|\nabla S_{0}(x)\right|^{2}}
\end{array}\right)\left(\mathfrak{G}_{H}^{s} c_{0}\right)(x) d s d \tau
$$

The right side equals

$$
\begin{aligned}
e^{i S_{0}(x)} & \int d s \delta(s)\left(\begin{array}{cc}
\mathbf{1}_{n}-\frac{\nabla S_{0}(x) \otimes \nabla S_{0}(x)}{\left|\nabla S_{0}(x)\right|^{2}} & 0_{n} \\
0_{n} & \mathbf{1}_{n}-\frac{\nabla S_{0}(x) \otimes \nabla S_{0}(x)}{\left|\nabla S_{0}(x)\right|^{2}}
\end{array}\right)\left(\mathfrak{G}_{H}^{s} c_{0}\right)(x) \\
= & e^{i S_{0}(x)}\left(\begin{array}{cc}
\mathbf{1}_{n}-\frac{\nabla S_{0}(x) \otimes \nabla S_{0}(x)}{\left|\nabla S_{0}(x)\right|^{2}} & 0_{n} \\
0_{n} & \mathbf{1}_{n}-\frac{\nabla S_{0}(x) \otimes \nabla S_{0}(x)}{\left|\nabla S_{0}(x)\right|^{2}}
\end{array}\right) c_{0}(x) \\
= & e^{i S_{0}(x)} c_{0}(x),
\end{aligned}
$$

since $\left(c_{0 \pm}, \nabla S_{0}(x)\right)=0$ on supp $c_{0}(x)$. Differentiating the right side of (9.3) yields:

$$
\begin{aligned}
& \dot{B}_{ \pm}=-(U \mp H, \nabla) e^{i S} \frac{1}{2 \pi} \int \cdots d s d \tau \\
&+\frac{e^{i S}}{2 \pi} \int e^{-i \tau s} \frac{\partial a}{\partial t} \cdot\left(\mathfrak{G}_{U}^{t} \mathfrak{G}_{H}^{s} c_{0}\right)(x) d s d \tau \\
&+\frac{e^{i S}}{2 \pi} \int e^{-i \tau s} a(x, \nabla S, t, \tau)\left[-\left(U, \nabla_{x}\right)\right]\left(\mathfrak{G}_{U}^{t} \mathfrak{G}_{H}^{s} c_{0}\right)(x) d s d \tau \\
&\left.+\frac{e^{i S}}{2 \pi} \int e^{-i \tau s} \sum_{\alpha=1}^{n}\left(\begin{array}{l}
\frac{\partial a_{+}}{\partial \xi_{\alpha}}\left[-(U-H, \nabla) \frac{\partial S}{\partial x_{\alpha}}-\sum_{\beta=1}^{n} \frac{\partial(U-H)_{\beta}}{\partial x_{\alpha}} \frac{\partial S}{\partial x_{\beta}}\right. \\
\frac{\partial \xi_{\alpha}}{\partial x_{\alpha}}-(U+H, \nabla) \frac{\partial S}{\partial x_{\alpha}}-\sum_{\beta=1}^{n} \frac{\partial(U+H)_{\beta}}{\partial x_{\alpha}} \frac{\partial S}{\partial x_{\beta}}
\end{array}\right]\right) \\
&\left(\mathfrak{G}_{U}^{t} \mathfrak{G}_{H}^{s} c_{0}\right)(x) d s d \tau .
\end{aligned}
$$


Using (4.12) we obtain

$$
\begin{aligned}
& \dot{B}_{ \pm}=-(U \mp H, \nabla) e^{i S} \frac{1}{2 \pi} \int \cdots d s d \tau \\
& +\frac{e^{i S}}{2 \pi} \int e^{-i \tau S}\left(\begin{array}{l}
-(U-H, \nabla) a_{+}+\sum_{\alpha=1}^{n} \frac{\partial \alpha_{+}}{\partial \xi_{\alpha}} \sum_{\beta=1}^{n} \frac{\partial(U-H)_{\beta}}{\partial x_{\alpha}} \frac{\partial S}{\partial x_{\beta}}-i \tau a_{+} \\
-(U+H, \nabla) a_{-}+\sum_{\alpha=1}^{n} \frac{\partial a_{-}}{\partial \xi_{\alpha}} \sum_{\beta=1}^{n} \frac{\partial(U+H)_{\beta}}{\partial x_{\alpha}} \frac{\partial S}{\partial x_{\beta}}+i \tau a_{+}
\end{array}\right. \\
& \begin{array}{l}
\left.-\frac{\partial(U+H)}{\partial x} a_{-}+\frac{\nabla S \otimes \nabla S}{|\nabla S|^{2}}\left(\frac{\partial(U-H)}{\partial x} a_{+}+\frac{\partial(U+H)}{\partial x} a_{-}\right)\right)\left(\mathfrak{G}_{U}^{t} \mathfrak{G}_{H}^{s} c_{0}\right) d s d \tau \\
\left.-\frac{\partial(U-H)}{\partial x} a_{+}+\frac{\nabla S \otimes \nabla S}{|\nabla S|^{2}}\left(\frac{\partial(U-H)}{\partial x} a_{+}+\frac{\partial(U+H)}{\partial x} a_{-}\right)\right)
\end{array} \\
& +\frac{e^{i S}}{2 \pi} \int e^{-i \tau s} a(x, \nabla S, t, \tau)\left[-\left(U, \nabla_{x}\right)\right]\left(\mathfrak{G}_{U}^{t} \mathfrak{G}_{H}^{s} c_{0}\right)(x) d s d \tau \\
& \left.+\frac{e^{i S}}{2 \pi} \int e^{-i \tau s} \sum_{\alpha=1}^{n}\left(\begin{array}{l}
\frac{\partial a_{+}}{\partial \xi_{\alpha}} \\
\frac{\partial a_{-}}{\partial \xi_{\alpha}}\left[-(U-H, \nabla) \frac{\partial S}{\partial x_{\alpha}}-\sum_{\beta=1}^{n} \frac{\partial(U-H)_{\beta}}{\partial x_{\alpha}} \frac{\partial S}{\partial x_{\beta}}\right. \\
-(U+H, \nabla) \frac{\partial S}{\partial x_{\alpha}}-\sum_{\beta=1}^{n} \frac{\partial(U+H)_{\beta}}{\partial x_{\alpha}} \frac{\partial S}{\partial x_{\beta}}
\end{array}\right]\right) \\
& \left(\mathfrak{G}_{U}^{t} \mathfrak{G}_{H}^{s} c_{0}\right)(x) d s d \tau \text {. }
\end{aligned}
$$

Integrating by parts the term $\mp i \tau a_{ \pm}$simplifies the expression as follows:

$$
\begin{aligned}
\int e^{i \tau s} & \left(\mp i \tau a_{ \pm}\right)\left(\mathfrak{G}_{U}^{t} \mathfrak{G}_{H}^{s} c_{0}\right)(x) d s d \tau \\
= & \pm \int \frac{d}{d s}\left(e^{-i \tau s}\right) a_{ \pm}(x, \nabla S, t, \tau)\left(\mathfrak{G}_{U}^{t} \mathfrak{G}_{H}^{s} c_{0}\right)(x) d s d \tau \\
= & \mp \int e^{-i \tau s} a_{ \pm}\left(\mathfrak{G}_{U}^{t} \frac{d}{d s} \mathfrak{G}_{H}^{s} c_{0}\right)(x) d s d \tau \\
& = \pm \int e^{-i \tau s} a_{ \pm} \cdot\left[\left(H, \nabla_{x}\right)\right]\left(\mathfrak{G}_{U}^{t} \mathfrak{G}_{H}^{s} c_{0}\right)(x) d s d \tau
\end{aligned}
$$

Combining (9.4) and (9.5) yields

$$
\begin{aligned}
\dot{B}_{ \pm}= & -(U \mp H, \nabla) B_{ \pm}-\frac{\partial(U \pm H)}{\partial x} B_{\mp} \\
& +\frac{\nabla S \otimes \nabla S}{|\nabla S|^{2}}\left(\frac{\partial(U-H)}{\partial x} B_{+}+\frac{\partial(U+H)}{\partial x} B_{-}\right) .
\end{aligned}
$$

We used here the fact that for all $t$

$$
-\left(H(x), \nabla_{x}\right) \nabla_{x} S(x, t)-\left(\frac{\partial H(x)}{\partial x}\right)^{\mathrm{T}} \nabla_{x} S(x, t)=0 .
$$

We thus established that $B_{ \pm}(x, t)$ defined as the right side of (9.3) satisfies both the equation and the initial condition (9.2). The uniqueness theorem for such a Cauchy problem implies the statement of Lemma 9.1.

REMARK 9.2. Let $J_{0}(x)$ be defined as follows:

$$
\begin{gathered}
(H, \nabla) J_{0}(x)=1, \\
\dot{J}(x, t)=-(U(x), \nabla) J
\end{gathered}
$$


Let $B=\left(\begin{array}{l}B_{+} \\ B_{-}\end{array}\right)$be a solution to $(9.2)$. Then $B e^{i J(x, t) \tau_{0}}$ is a solution to

$$
\begin{aligned}
\dot{B}_{ \pm}= & -(U \mp H, \nabla) B_{ \pm} \pm i \tau_{0} B_{ \pm} \\
& -\frac{\partial(U \pm H)}{\partial x} B_{\mp}+\frac{\nabla S \otimes \nabla S}{|\nabla S|^{2}}\left(\frac{\partial(U-H)}{\partial x} B_{+}+\frac{\partial(U+H)}{\partial x} B_{-}\right) .
\end{aligned}
$$

10. Instability criterion. Let $F$ be a magnetic field line for $H$. Let $\mathcal{U}$ be an open neighborhood stretched along $F$ and let $b_{0 \pm}(x)$ be a $C_{0}^{\infty}$-vector field such that $\operatorname{supp} b_{0 \pm} \subset \mathcal{U}$. Let $S_{0}(x)$ be defined in $\mathcal{U}$ and satisfy the following conditions: $(H(x)$, $\left.\nabla S_{0}(x)\right)=0,\left(b_{0 \pm}(x), \nabla S_{0}(x)\right)=0$, and $|\nabla S(x)| \geq c_{0}>0$ for all $x$ in $\mathcal{U}$. We define the test function

$$
\varphi_{0 \pm}(x)=i \varepsilon \nabla \times\left\{\frac{\nabla S_{0} \times b_{0 \pm}(x)}{\left|\nabla S_{0}\right|^{2}} e^{i S_{0}(x) / \varepsilon}\right\}
$$

It follows from (10.1) that

$$
\varphi_{0 \pm}(x)=b_{0 \pm}(x) e^{i S_{0}(x) / \varepsilon}+O(\varepsilon)
$$

where the constant in $O$ is uniform with $x \in \mathcal{U}$.

We compute $G(t) \varphi_{0}$ up to the terms of order $\sqrt{\varepsilon}$. According to Theorem 7.1

$$
\| G(t) \circ \text { op }[1-\chi(\sqrt{\varepsilon} \xi)] \varphi_{0}-G_{\varepsilon}^{s}(t) \varphi_{0}\left\|_{\mathcal{L}^{2}} \leq C \sqrt{\varepsilon}\right\| \varphi_{0} \|_{D(\Lambda)} .
$$

We estimate the term op $[\chi(\sqrt{\varepsilon} \xi)] \varphi_{0}$ on the left side of $(10.3)$.

LEMMA 10.1. For any $N \geq 1$

$$
\left\|\operatorname{op}[\chi(\sqrt{\varepsilon} \xi)] \varphi_{0}\right\|_{\mathcal{L}^{2}} \leq C \varepsilon^{N}\left\|b_{0}\right\|_{W^{N+1,2}(\mathcal{U})} .
$$

Proof. We have

$$
\begin{aligned}
\operatorname{op}[\chi(\sqrt{\varepsilon} \xi)] \varphi_{0 \pm}= & \frac{i \varepsilon}{(2 \pi \sqrt{\varepsilon})^{n}} \int e^{i \xi(x-y) / \sqrt{\varepsilon}} \chi(\xi) \nabla \times\left(\frac{\nabla S_{0} \times b_{0 \pm}(y)}{|\nabla S|^{2}} e^{i S(y) / \varepsilon}\right) d y d \xi \\
= & \frac{1}{(2 \pi \sqrt{\varepsilon})^{n}} \int e^{i \xi(x-y) / \sqrt{\varepsilon}+i\left(S_{0}(y) / \varepsilon\right)} \chi(\xi) \\
& \cdot\left(b_{0 \pm}(y)+i \varepsilon \nabla \times\left(\frac{\nabla S_{0} \times b_{0 \pm}(y)}{|\nabla S|^{2}}\right)\right) d y d \xi .
\end{aligned}
$$

Let

$$
L=i \sum_{j=1}^{n}\left(\sqrt{\varepsilon} \xi_{j}-\nabla_{j} S_{0}(y)\right) \frac{\partial}{\partial y_{j}} .
$$

Then

$$
\begin{aligned}
\mathrm{op}[\chi(\sqrt{\varepsilon} \xi) \varphi](x)= & \frac{1}{(2 \pi \sqrt{\varepsilon})^{n}} \int \frac{\varepsilon \chi(\xi)}{\left|\sqrt{\varepsilon} \xi-\nabla S_{0}(y)\right|^{2}} L\left[e^{i \xi(x-y) / \sqrt{\varepsilon}+i\left(S_{0}(y) / \varepsilon\right)}\right] \\
& \cdot\left(b_{0 \pm}(y)+i \varepsilon \nabla \times\left(\frac{\nabla S_{0}(y) \times b_{0 \pm}(y)}{\left|\nabla S_{0}(y)\right|^{2}}\right)\right) d y d \xi
\end{aligned}
$$

Integrating by parts using the operator $L N$-times and then estimating the integral via the Calderon-Vaillancourt inequality yields the statement of the lemma. 
LEMMA 10.2 .

$$
\left\|\varphi_{0}\right\|_{D(\Lambda)} \leq C\left(\left\|b_{0}\right\|_{W^{1,2}}+\varepsilon\left\|b_{0}\right\|_{W^{2,2}}\right)
$$

Proof. We have

$$
\begin{aligned}
\left\|\varphi_{0 \pm}\right\|_{D(\Lambda)} \leq \| & \varphi_{0 \pm}\left\|_{L^{2}(\mathcal{U})}+\right\|(H, \nabla) \varphi_{0 \pm} \|_{L^{2}(\mathcal{U})} \\
\leq & \left\|b_{0 \pm}\right\|_{L^{2}(\mathcal{U})}+C \varepsilon\left\|b_{0 \pm}\right\|_{W^{1,2}(\mathcal{U})} \\
& \quad+\frac{1}{\varepsilon}\left\|\left(H, \nabla S_{0}\right)\left(b_{0 \pm}+i \varepsilon \nabla \times\left(\frac{\nabla S_{0} \times b_{0 \pm}}{\left|\nabla S_{0}\right|^{2}}\right)\right)\right\|_{L^{2}(\mathcal{U})} \\
& +\left\|(H, \nabla)\left(b_{0 \pm}+i \varepsilon \nabla \times\left(\frac{\nabla S_{0} \times b_{0 \pm}}{\left|\nabla S_{0}\right|^{2}}\right)\right)\right\|_{L^{2}(\mathcal{U})} .
\end{aligned}
$$

The third term in the right side vanishes since $\left(H(y), \nabla S_{0}(y)\right)=0$ for $y \in \mathcal{U}$. The statement of the lemma now follows from (10.6).

It follows from (10.3), (10.4), and (10.5) $[N=1]$ that

$$
\left\|G(t) \varphi_{0}-G_{\varepsilon}^{s}(t) \varphi_{0}\right\|_{\mathcal{L}^{2}} \leq C\left(\sqrt{\varepsilon}\left\|b_{0}\right\|_{W^{1,2}(\mathcal{U})}+\varepsilon\left\|b_{0}\right\|_{W^{2,2}(\mathcal{U})}\right) .
$$

TheOREM 10.3. Let $t \in[0, T]$. Then there exists a constant $M=M(n)$ such that

$$
\left\|G_{\varepsilon}^{s}(t) \varphi_{0}-e^{i S(x, t) / \varepsilon} B(x, t)\right\|_{\mathcal{L}^{2}} \leq C \sqrt{\varepsilon}\left\|b_{0}\right\|_{W^{M, 2}} .
$$

Here the amplitude $B(x, t)$ satisfies $(9.2)$ with $c_{0}(x)=b_{0}(x)$.

Proof. Using (7.5) and Theorem 6.1 we conclude that

$$
\left\|G_{\varepsilon}^{s}(t) \varphi_{0}-\mathrm{op}_{H}[A(t)] \varphi_{0}\right\|_{\mathcal{L}^{2}} \leq C \sqrt{\varepsilon}\left\|\varphi_{0}\right\|_{D(\Lambda)} \leq C \sqrt{\varepsilon}\left\|b_{0}\right\|_{W^{2,2}(\mathcal{U})} .
$$

In addition to this, from (10.6) and Theorem 6.1

$$
\left\|\mathrm{op}_{H}[A(t)]\left(\varphi_{0}-\left(b_{0} e^{i S_{0} / \varepsilon}\right)\right)\right\|_{\mathcal{L}^{2}} \leq C \varepsilon\left\|b_{0}\right\|_{W^{2,2}(\mathcal{U})} .
$$

The constant $C$ in the right sides of (10.9), (10.10) is uniform with respect to $t \in[0, T]$. It follows from (10.9), (10.10) that

$$
\left\|G_{\varepsilon}^{s}(t) \varphi_{0}-\mathrm{op}_{H}[A(t)]\left(b_{0} e^{i S_{0} / \varepsilon}\right)\right\|_{\mathcal{L}^{2}} \leq C \sqrt{\varepsilon}\left\|b_{0}\right\|_{W^{2,2}(\mathcal{U})} .
$$

We now have to compute op ${ }_{H}[A(t)]\left(b_{0} e^{i S / \varepsilon}\right)$ up to a small error. We proceed as follows: for any $N \in Z_{+}$

$$
\begin{aligned}
\mathrm{op}_{H} & {[A(t)]\left(b_{0} e^{i S_{0} / \varepsilon}\right) } \\
& =\frac{1}{2 \pi} \int e^{-i s \tau} \mathrm{op}_{\varepsilon}[A(t, \tau)]\left\{e^{i S(t) / \varepsilon} \mathfrak{G}_{U}^{t} \mathfrak{G}_{H}^{s} b_{0}\right\} d s d \tau \\
& =\frac{1}{2 \pi} \int(1-i \tau)^{-N}\left\{\left(1+\frac{d}{d s}\right)^{N} e^{-i s \tau}\right\} \mathrm{op}_{\varepsilon}[A(t, \tau)]\left\{e^{i S(t) / \varepsilon} \mathfrak{G}_{U}^{t} \mathfrak{G}_{H}^{s} b_{0}\right\} d s d \tau \\
& =\frac{1}{2 \pi} \int(1-i \tau)^{-N} e^{-i s \tau} \chi\left(\frac{s}{t+1}\right) \operatorname{op}^{\varepsilon}[A(t, \tau)]\left\{e^{i S(t) / \varepsilon} \mathfrak{G}_{U}^{t} \mathfrak{G}_{H}^{s}(i d+(H, \nabla))^{N} b_{0}\right\} d s d \tau
\end{aligned}
$$

Here $\chi \in C_{0}^{\infty}(\mathbb{R})$ is chosen as in the proof of Theorem 6.1. Since the integral in $\tau$ is absolutely convergent for $N \geq 2$, we will use a variant of the stationary phase formula applied in Vishik [16]. 
Lemma 10.4. Let $S \in \mathcal{D}\left(\mathbb{R}^{n}\right), B \in S_{0,0}^{0}$. There exists a constant $C=C(n, B, S)$ depending on a certain number of derivatives of $S$ and the seminorms of $B$ in $S_{0,0}^{0}$ and a constant $K=K(n)$ such that for any $v_{0} \in \mathcal{D}\left(\mathbb{R}^{n}\right)$

$$
\left|\left\{\operatorname{op}_{\varepsilon}[B]\left(e^{i S / \varepsilon} v_{0}\right)\right\}(x)-B(x, \nabla S(x)) e^{i S(x) / \varepsilon} v_{0}(x)\right| \leq C \varepsilon \sum_{|\alpha| \leq K} \sup _{y \in \mathbb{R}^{n}}\left|\frac{\partial^{\alpha}}{\partial y^{\alpha}} v_{0}(y)\right|, x \in \mathbb{R}^{n}
$$

Proof. See Lemma 9.1 in [16].

Lemma 10.5. Let $B \in S_{0,0}^{0}$ and $v_{0} \in \mathcal{D}\left(\mathbb{R}^{n}\right)$ so that $\operatorname{supp} v_{0} \subset \Omega \subset \subset \mathbb{R}^{n}$, and $d(\operatorname{supp} w$, $\left.\mathbb{R}^{n} \backslash \Omega\right) \geq 1$. Then for any $Q \in \mathbb{Z}_{+}$

$$
\left\|\mathrm{op}_{\varepsilon}[B] w\right\|_{L^{2}\left(\mathbb{R}^{n} \backslash \Omega\right)} \leq C_{Q} \varepsilon^{Q}\|w\|_{L^{2}\left(\mathbb{R}^{n}\right)} .
$$

Proof. See Lemma 9.2 in [16].

Extending $S$ by zero outside some open set containing $U$ we can apply Lemmas 10.4 and 10.5 to the integral in the right side of (10.12). It is clear that the constants are uniformly bounded with $t \in[0, T]$. Thus

$$
\begin{gathered}
\|\left\{\operatorname{op}_{H}[A(t)] b_{0} e^{i S_{0} / \varepsilon}\right\}(x) \\
\quad-\frac{1}{2 \pi} \int(1-i \tau)^{-N} e^{-i \tau s} \chi\left(\frac{s}{t+1}\right) A(x, \nabla S(x, t), t, \tau) e^{i S(x, t) / \varepsilon} \\
\quad \cdot\left(\mathfrak{G}_{U}^{t} \mathfrak{G}_{H}^{s}(i d+(H, \nabla))^{N} b_{0}\right)(x) d s d \tau \|_{L^{2}} \\
\leq C \varepsilon\left\|b_{0}\right\|_{C^{K+N}} .
\end{gathered}
$$

We next integrate by parts with respect to $s$ to obtain

$$
\begin{gathered}
\|\left\{\mathrm{op}_{H}[A(t)] b_{0} e^{i S_{0} / \varepsilon}\right\}(x)-\frac{e^{S(x, t) / \varepsilon}}{2 \pi} \int e^{-i \tau s} A(x, \nabla S(x, t), t, \tau) \\
\cdot\left(\mathfrak{G}_{U}^{t} \mathfrak{G}_{H}^{s} b_{0}\right)(x) d s d \tau\left\|_{L^{2}} \leq C \varepsilon\right\| b_{0} \|_{W^{K+N+[n / 2]+1,2}} .
\end{gathered}
$$

We have used the Sobolev embedding theorem in the last step. Obviously (10.14), (10.11) together with Lemma 9.1 imply (10.8). We remark that $A(x, \nabla S(x, t), t, \tau) \equiv$ $a(x, \nabla S(x, t), t, \tau), t \in[0, T]$, for $\varepsilon$ sufficiently small, since $\left|\nabla S_{0}\right| \geq c_{0}>0$. This concludes the proof of Theorem 10.3 .

To formulate the instability criterion we consider the system

$$
\left\{\begin{array}{l}
\dot{B}_{ \pm}(x, \xi, t)=-(U \mp H, \nabla) B_{ \pm} \pm i \tau_{0} B_{ \pm} \\
-\frac{\partial(U \pm H)}{\partial x} B_{\mp}+\frac{\xi \otimes \xi}{|\xi|^{2}}\left(\frac{\partial(U-H)}{\partial x} B_{+}+\frac{\partial(U+H)}{\partial x} B_{-}\right) \\
\left(B_{ \pm}, \xi\right)=0 ; \quad B_{ \pm}(x, \xi, 0)=B_{ \pm 0}(x, \xi) .
\end{array}\right.
$$

Let $F$ be a magnetic field line and let $\widetilde{F}$ be a trajectory in the cotangent bundle with removed zero section $(x, \xi) \in R^{n} \times\left(\left(R^{n}\right)^{*} \backslash 0\right)$ covering $F: \frac{d x}{d s}=H(x) ; \frac{d \xi}{d s}=-\left(\frac{\partial H}{\partial x}\right)^{\mathrm{T}} \xi$. 
According to Huygens' Principle the system (10.15) could be solved on each $\widetilde{F}$, i.e., the domain of dependence for $(x, \xi) \in \widetilde{F}$ is

$$
\bigcup_{s=-t}^{t} \mathfrak{G}_{U}^{-t} \mathfrak{G}_{H}^{-s}(x, \xi) \subset \mathfrak{G}_{U}^{-t} \widetilde{F}
$$

Therefore we could prescribe the data $B_{ \pm}(x, \xi, 0)$ for $(x, \xi) \in \widetilde{F}$. Denote by $L^{2}(\widetilde{F})$ the space of all data $B_{0}=\left(\begin{array}{c}B_{0+}(x, \xi) \\ B_{0-}(x, \xi)\end{array}\right),(x, \xi) \in \widetilde{F}$,

$$
\left(B_{0 \pm}(x, \xi), \xi\right)=0, \quad \int d s|B(x(s), \xi(x))|^{2}=\int_{F}|B(x, \xi(x))|^{2} \frac{|d x|}{|H(x)|} .
$$

Then the solution to (10.15) defines an operator

$$
\mathfrak{G}_{F}(t): L^{2}(\widetilde{F}) \rightarrow L^{2}\left(g_{U}^{t} \widetilde{F}\right) .
$$

THEOREM 10.6. For any nonperiodic trajectory $F$ and any covering trajectory $\widetilde{F}$ in $T^{*} R^{n} \backslash\{0\}$ and for any real $\tau_{0}$

$$
\lim _{t \rightarrow \infty} \frac{1}{t} \log \|G(t)\|_{\mathcal{L}_{s}^{2}} \geq \varlimsup_{t \rightarrow \infty} \frac{1}{t} \log \left\|\mathfrak{G}_{F}(t)\right\|_{\mathcal{L}\left(L^{2}(\tilde{F}), L^{2}\left(g_{U}^{t}(\tilde{F})\right)\right.}
$$

Proof. We choose an arbitrary smooth $v_{0} \in \mathcal{D}\left(\mathbb{R}^{n}\right)$. Let $D_{\delta}\left(x_{0}\right)$ be a disc of radius $\delta$ perpendicular to $H\left(x_{0}\right)$ at $x_{0}$. Let $\xi_{\delta}(x)$ be constructed as follows: $\left.\xi_{\delta}\right|_{D_{\delta}\left(x_{0}\right)} \in \mathcal{D}\left(D_{\delta}\left(x_{0}\right)\right)$ is a cutoff function: $\xi_{\delta} \geq 0 ;\left.\xi_{\delta}\right|_{\delta / 2\left(x_{0}\right)} \equiv 1 ; \xi_{\delta}(x)=\xi_{1}\left(x_{0}+\frac{x-x_{0}}{\delta}\right), x \in D_{\delta}\left(x_{0}\right),(H, \nabla) \xi_{\delta}=$ 0 . For sufficiently small $\delta, \xi_{\delta}$ is well defined at least on $\operatorname{supp} v_{0}$ since $F$ is nonperiodic.

We now set

$$
b_{0}^{(\delta)}=v_{0}(x) \cdot \xi_{\delta}(x)
$$

Let $\xi_{\delta}(x, t)$ satisfy

$$
\dot{\xi}_{\delta}(x, t)=-(U, \nabla) \xi_{\delta}, \quad \xi_{\delta}(x, 0)=\xi_{\delta}(x) .
$$

We solve the system (9.2) with the initial condition

$$
B(x, 0)=v_{0}(x) \cdot \xi_{\delta}(x) \cdot e^{i S_{0}(x) / \varepsilon} .
$$

The solution is given by $B_{ \pm}(x, t) \cdot \xi_{\delta}(x, t)$. We compute the $\|\cdot\|_{\mathcal{L}^{2}}$ of the right-hand side of (10.19):

$$
\begin{aligned}
\left\|v_{0}(x) \xi_{\delta}(x) e^{i S_{0}(x) / \varepsilon}\right\|_{\mathcal{L}^{2}} & \\
= & \left(\int\left|v_{0}(x)\right|^{2}\left|\xi_{\delta}(x)\right|^{2} d x\right)^{1 / 2} \\
= & \left.\left.\left|\int_{D_{\delta}\left(x_{0}\right)}\right| \xi_{\delta}(x)\right|^{2} d x\right|^{1 / 2}\left\{\left(\int_{-\infty}^{\infty}\left|v_{0}(x(s))\right|^{2} d s\right)^{1 / 2}+o(\delta)\right\} \\
= & c \delta\left(\int_{-\infty}^{\infty}\left|v_{0}(x(s))\right|^{2} d s\right)^{1 / 2}+o(\delta),
\end{aligned}
$$


where

$$
c=\left(\int_{D_{1}\left(x_{0}\right)}\left|\xi_{1}(x)\right|^{2} d^{n-1} x\right)^{1 / 2}
$$

According to Theorem 10.3 and (10.7),

$$
\left\|G(t) \varphi_{0}-e^{i S(x, t) / \varepsilon} \xi_{\delta}(x, t) B(x, t)\right\|_{\mathcal{L}^{2}} \leq c \sqrt{\varepsilon}\left\|v_{0} \xi_{\delta}(x)\right\|_{W^{M, 2}} .
$$

A computation analogous to (10.20) yields

$$
\left\|B(x, t) \xi_{\delta}(x, t) e^{i S(x, t) / \varepsilon}\right\|_{\mathcal{L}^{2}}=c \delta\left(\int_{g_{U}^{t} F}|B(x(s), t)|^{2} d s\right)^{1 / 2}+o(\delta)
$$

Thus,

$$
\left\|G(t) \varphi_{0}\right\|_{\mathcal{L}^{2}} \geq c \delta\left(\int_{g_{U}^{t} F}|B(x(s), t)|^{2} d s\right)^{1 / 2}+o_{\delta \rightarrow 0}(\delta)-C \sqrt{\varepsilon}\left\|v_{0} \xi_{\delta}(x)\right\|_{W^{M, 2}} .
$$

Hence,

$$
\left\|G(t) \varphi_{0}\right\|_{\mathcal{L}^{2}} /\left\|\varphi_{0}\right\|_{\mathcal{L}^{2}} \geq \frac{\left(\int_{g_{U}^{t} F}|B(x(s), t)|^{2} d s\right)^{1 / 2}+o_{\delta \rightarrow 0}(1)-O\left(\sqrt{\varepsilon} \delta^{-1}\right)}{\left(\int_{F}\left|v_{0}(x(s))\right|^{2} d s\right)^{1 / 2}-o_{\delta \rightarrow 0}(1)+O\left(\varepsilon \delta^{-1}\right)} .
$$

As $\varepsilon \rightarrow 0$ we get

$$
\|G(t)\|_{\mathcal{L}_{s}^{2}} \geq \frac{\left(\int_{g_{U}^{t} F}|B(x(s), t)|^{2} d s\right)^{1 / 2}+o_{\delta \rightarrow 0}(1)}{\left(\int_{F}\left|v_{0}(x(s))\right|^{2} d s\right)^{1 / 2}-o_{\delta \rightarrow 0}(1)}
$$

As $\delta \rightarrow 0$ we obtain

$$
\|G(t)\|_{\mathcal{L}_{s}^{2}} \geq \frac{\left(\int_{g_{U}^{t} F}|B(x(s), t)|^{2} d s\right)^{1 / 2}}{\left(\int_{F}\left|v_{0}(x(s))\right|^{2} d s\right)^{1 / 2}} .
$$

Since the choice of $S_{0}$ satisfying the conditions at the beginning of $\S 9$ was arbitrary, this provides arbitrary $\xi_{0}=\nabla S\left(x_{0}\right)$ which gives arbitrary $\widetilde{F}$ covering $F$. We may assume without any loss of generality that $\left|\xi_{0}\right|=1$. We refer to Remark 9.2 to complete the proof.

In the case of closed magnetic field lines it is necessary to modify the construction to ensure that $S$ is well defined. This results in a quantization condition that restricts the parameter $\tau \in 2 \pi \mathbb{Z} / T_{0}$ where $T_{0}$ is the period of the closed stream line.

REMARK. In the case where there exists a singular point $x_{0}$ such that $H\left(x_{0}\right)=0$ the instability criterion is augmented by the following lower bound:

$$
\lim _{t \rightarrow \infty} \frac{1}{t} \log \|G(t)\|_{\mathcal{L}_{s}^{2}} \geq \lim _{t \rightarrow \infty} \frac{1}{t} \log \sup _{\substack{x_{0}, \xi_{0}, b_{ \pm 0} \\\left|\xi_{0}\right|=1,\left|b_{+_{0}}\right|^{2}+\left|b_{-0}\right|^{2}=1 \\\left(\xi_{0}, b_{ \pm_{0}}\right)=0 \\ H\left(x_{0}\right)=0}}\left|b_{ \pm}(t)\right|
$$


where $x, \xi$, and $b_{ \pm}$satisfy the following system and the $O D E$

$$
\left\{\begin{array}{l}
\frac{d x}{d t}=U(x), \\
\frac{d \xi}{d t}=-\left(\frac{\partial U}{\partial x}\right)^{\mathrm{T}} \xi \\
\frac{d b_{ \pm}}{d t}=-\frac{\partial(U \pm H)}{\partial x} b_{\mp}+\left[\left(\frac{\partial(U+H)}{\partial x} b_{-}+\frac{\partial(U-H)}{\partial x} b_{+}\right) \frac{\xi \otimes \xi}{|\xi|^{2}}\right.
\end{array}\right.
$$

with the initial conditions

$$
x(0)=x_{0}, \quad \xi(x, 0)=\xi_{0} \in \mathbb{R}^{n} \backslash 0, \quad b(x, 0)=b_{0} \in \mathbb{R}^{2 n} .
$$

The proof of this result is exactly analogous to the proof for the fluid problem given in [16].

Acknowledgments. S. Friedlander thanks MSRI, Berkeley for its kind hospitality and acknowledges support from NSF grants DMS 95-00466 and DMS 96-22563. M. Vishik gratefully acknowledges support from DSM 93-01172 and DMS 95-31769. Their collaborative work is partially supported by DMS 93-00752.

\section{REFERENCES}

[1] L. Boutet de Monvel, Hypoelliptic operators with double characteristics, Comm. Pure Appl. Math. 27, 585-639 (1974)

[2] A. Calderon and R. Vaillancourt, A class of bounded pseudodifferential operators, Proc. Nat. Acad. Sci. USA 69, 1185-1187 (1972)

[3] S. Chandrasekhar, Hydrodynamic and Hydromagnetic Stability, Dover, 1981

[4] J. Chazarain and A. Piriou, Introduction to the Theory of Linear Partial Differential Equations, North-Holland Publishing Co., Amsterdam, New York, 1982

[5] R. Coifman and Y. Meyer, Au delá des opérateurs pseudodifferentials, Asterisque 57, 1-185 (1978)

[6] K. S. Eckhoff, Linear waves and stability in ideal magnetohydrodynamics, Phys. Fluids 30, no. 12, 3673-3685 (1987)

[7] F. G. Friedlander, Sound Pulses, Cambridge University Press, 1958

[8] S. Friedlander and M. Vishik, Instability criteria for steady flows of a perfect fluid, Chaos 2 , no. 3, 455-460 (1992)

[9] S. Friedlander and M. Vishik, On stability and instability criteria for magnetohydrodynamics, Chaos 5, no. 2, 416-423 (1995)

[10] E. Hameiri, On the essential spectrum of ideal MHD, Comm. Pure Appl. Math. 38, 43-66 (1985)

[11] J. Hounie, On the $L^{2}$-continuity of pseudodifferential operators, Comm. Partial Differential Equations 11, no. 7, 765-778 (1986)

[12] A. E. Lifschitz, Continuous spectrum in general toroidal systems (ballooning and Alfven modes), Phys. Lett. 122A, 350-356 (1987)

[13] D. Ludwig, Exact and asymptotic solution of the Cauchy problem, Comm. Pure Appl. Math. 13, 473-508 (1960)

[14] E. Stein, Harmonic Analysis, Princeton University Press, 1994

[15] F. Treves, Introduction to Pseudodifferential and Fourier Integral Operators, Plenum Press, New York, London, 1980

[16] M. Vishik, Spectrum of small oscillations of an ideal fluid and Lyapunov exponents, J. Math. Pures Appl. 75, 531-557 (1996)

[17] M. Vishik and S. Friedlander, Dynamo theory methods for hydrodynamic stability, J. Math. Pures Appl. 72, 145-180 (1993) 